\title{
Clinical and Microbiological Characteristics of Candida guilliermondii and Candida fermentati
}

Tatsuro Hirayama ${ }^{1)}$, Taiga Miyazaki ${ }^{1)}$, Takahiro Takazono') ${ }^{1)}$ Tomomi Saijo ${ }^{1)}$, Shintaro Shimamura ${ }^{1)}$, Kazuko Yamamoto' $^{1)}$, Yoshifumi Imamura' $^{1)}$, Koichi Izumikawa ${ }^{2)}$, Katsunori Yanagihara ${ }^{3)}$, Shigeru Kohno ${ }^{1)}$, Hiroshi Mukae ${ }^{1)}$

1) Second Department of Internal Medicine 2) Infection Control and Education Center 3) Department of Laboratory Medicine, Nagasaki University Hospital, Japan

Objectives: The Candida guilliermondii complex is a genetically heterogeneous complex of several phenotypically indistinguishable species, including C. guilliermondii and Candida fermentati (1). C. guilliermondii and C. fermentati cannot be distinguished by the commercial phenotypic and biochemical methods used in clinical practice (2). This complex is characterized by decreased susceptibility to echinocandin and azole antifungals $(3,4)$, however, there have been limited studies reporting the epidemiological and clinical features of $C$. guilliermondii complex infections. This study aimed to clarify the clinical and microbiological characteristics of the $C$. guilliermondii complex.

Methods: Isolates were collected and re-identified by sequencing the internal transcribed spacer regions and restriction fragment length polymorphism of the intergenic spacer gene, confirming 27 C. guilliermondii and $12 \mathrm{C}$. fermentati isolates. Clinical information was reviewed retrospectively for all 39 patients. Susceptibility to eight antifungal agents was examined using a commercially prepared colorimetric microdilution panel, Sensititre YeastOne microtiter panel. Therapeutic effects of micafungin against $C$. guilliermondii clinical isolates were evaluated in a murine model of invasive candidiasis. We also explored differences in phenotype, including sensitivity to various stresses in vitro and resistance to candidacidal effect of murine macrophages, between $C$. guilliermondii and $C$. fermentati.

Results: Among all the clinical isolates, the majority $(81.5 \%, \mathrm{n}=22)$ of $C$. guilliermondii strains, but none of the $C$. fermentati strains, were isolated from blood cultures (Table 1). Most C. guilliermondii and all C. fermentati isolates were susceptible to both azoles and echinocandins when evaluated by the in vitro microdilution test (Table 2). However, micafungin was significantly less effective against $C$. guilliermondii than against Candida glabrata in a mouse model of disseminated candidiasis (Figure 2). Phenotypic analyses revealed that $C$. fermentati strains were more sensitive to hydrogen peroxide than the $C$. guilliermondii strains. In addition, murine RAW 264 macrophages had higher candidacidal effects against $C$. fermentati than against $C$. guilliermondii (Figure 1).

Conclusion: This study demonstrates that $C$. guilliermondii and $C$. fermentati are closely related but have different microbiological and clinical characteristics, highlighting the importance of the accurate identification of these species.

(A)
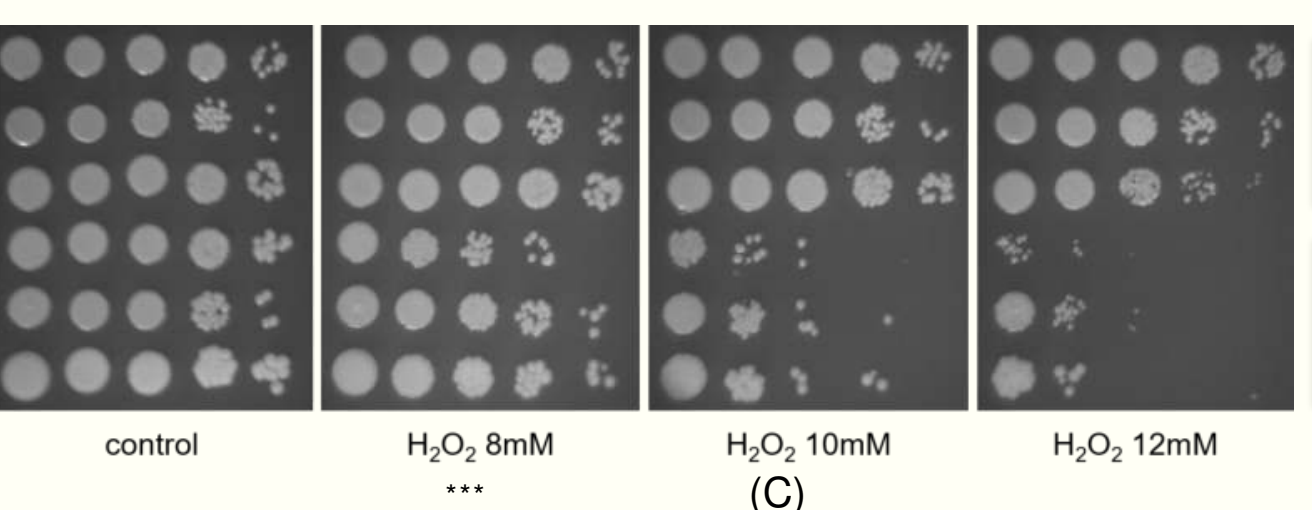

C. guilliermondi
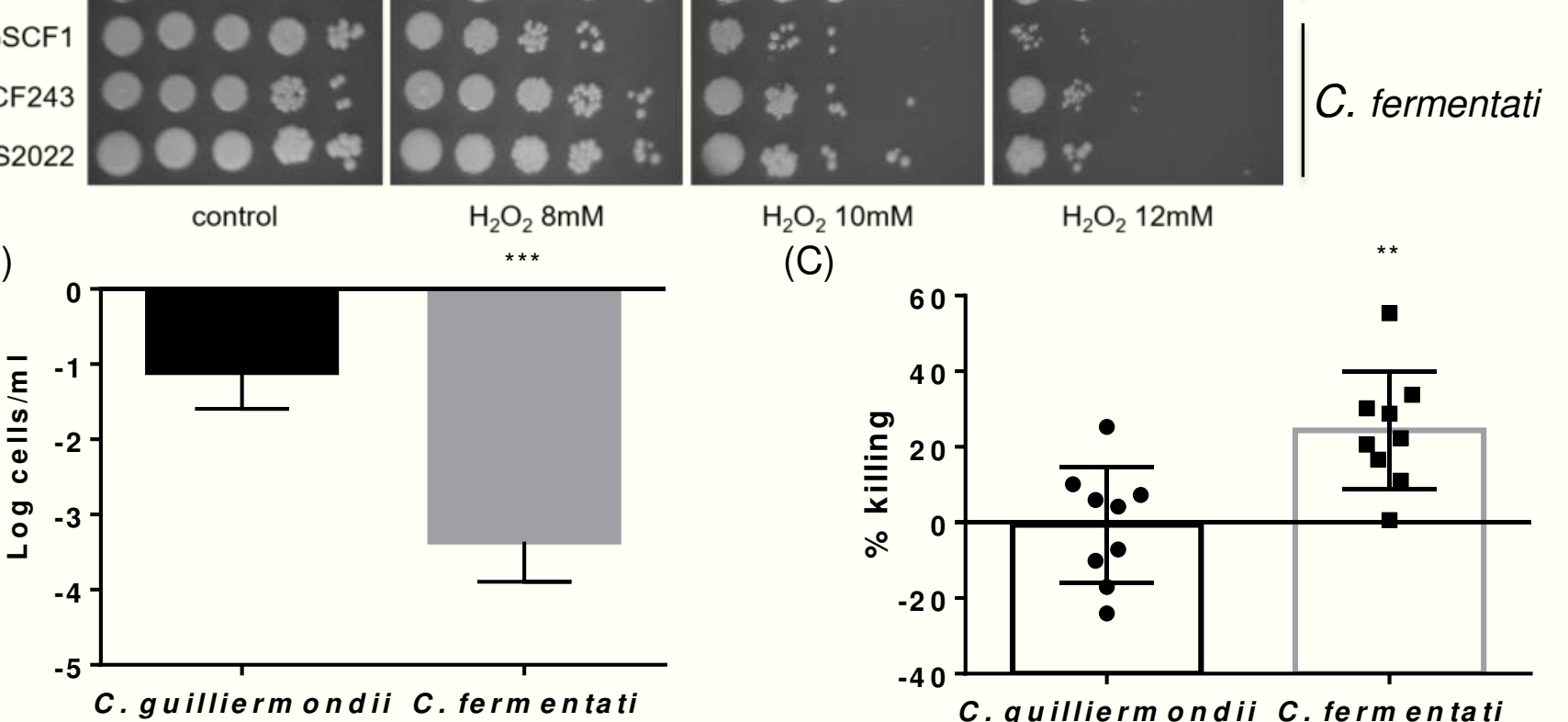

(C)

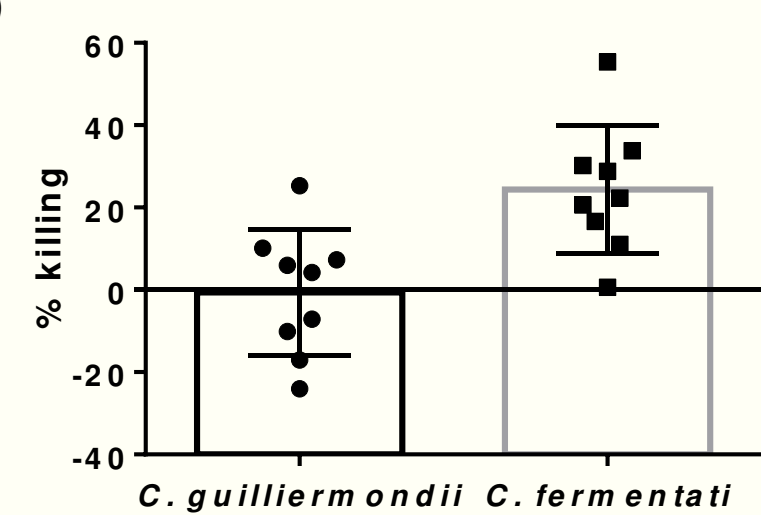

Figure 1. Growth and killing assay in the presence of $\mathrm{H}_{2} \mathrm{O}_{2}$ and killing assay by RAW 264. (A) Serial dilutions of logarithmic-phase cells of $C$. guilliermondii and $C$. fermentati strains were spotted onto SC plates containing $\mathrm{H}_{2} \mathrm{O}_{2}$ at the indicated concentrations. (B) Logarithmic-phase cells of $C$. guilliermondii and C. fermentati were treated with $5 \mathrm{mM} \mathrm{H}_{2} \mathrm{O}_{2}$ for $4 \mathrm{~h}$. The reduced viable cell counts of $C$. guilliermondii and $C$. fermentati were $1.1 \pm$ 0.4 and $3.4 \pm 0.5-\log$ cells $/ \mathrm{ml}$, respectively. There was a significant difference between the two groups (*** $P<0.001$ ). (C) Candida cell suspensions were co-cultured with RAW 264 cells at $37^{\circ} \mathrm{C}$ for $4 \mathrm{~h}$. Mean in vitro killing of $C$. guilliermondii and $C$. fermentati is expressed as the percent reduction of CFUs recovered from co-cultures compared with CFUs from control cultures (Candida cells without macrophages). The percent killing ratios of $C$. guilliermondii and $C$. fermentati were $-0.6 \pm 14.4 \%$ and $24.4 \pm$ $14.6 \%$, respectively. There was a significant difference between the two groups $(* * P<0.01)$.

\section{References}

(1) Vaughan-Martini A et al. FEMS Yeast Res.2005. (2) Lockhart SR et al. J Clin Microbiol.2009 (3) Chen CY et al. J Antimicrob Chemother. 2013. (4) Cheng JW et al. J Clin Microbiol. 2016
Table 1. Patient characteristics associated with $C$. guilliermondii complex isolates

\begin{tabular}{lccc}
\hline \multicolumn{1}{c}{ Characteristic } & C. guilliermondii $(n=27)$ & C. fermentati $(n=12)$ & $P$ value \\
\hline Age (yr), mean \pm SD & $63.3 \pm 18.7$ & $61.2 \pm 19.0$ & 0.75 \\
Male sex, no. (\%) & $20(74.1)$ & $7(58.3)$ & 0.46 \\
Co-malignancies, no. (\%) & $18(66.7)$ & $10(83.3)$ & 0.45 \\
Steroid use, no. (\%) & $11(40.7)$ & $8(66.7)$ & 0.18 \\
Central venous catheter, no. (\%) & $22(81.5)$ & $9(75.0)$ & 0.68 \\
In-hospital death, no. (\%) & $13(48.1)$ & $6(50.0)$ & 1.00 \\
Antifungal therapy, no. (\%) & $12(44.4)$ & $9(75.0)$ & 0.10 \\
Elevated $\beta-D$ glucan, no. (\%) & $\mathbf{1 6 ( 6 4 . 0 )}{ }^{\text {a }}$ & $\mathbf{2 ( 1 6 . 7 )}$ & $\mathbf{0 . 0 1}$ \\
Bloodstream isolates, no. (\%) & $\mathbf{2 2 ( 8 1 . 5 )}$ & $\mathbf{0 ( 0 )}$ & $<\mathbf{0 . 0 0 0 1}$ \\
Cause of infection, no. (\%) & $\mathbf{2 4 ( 8 8 . 9 )}$ & $\mathbf{0 ( 0 )}$ & $<\mathbf{0 . 0 0 0 1}$ \\
\hline
\end{tabular}

${ }^{a}$ Serum $\beta$-D glucan was measured for 25 patients.

Table 2. In vitro susceptibility of $27 C$. guilliermondii isolates and $12 C$. fermentati isolates to eight antifungal agents as interpreted by breakpoints for echinocandins and epidemiological cutoff values for other agents

\begin{tabular}{cccccccc}
$\begin{array}{c}\text { C. guilliermondii } \\
\text { complex (no. of } \\
\text { isolates) }\end{array}$ & \multicolumn{6}{c}{ MIC $(\mu \mathrm{g} / \mathrm{ml})$} & \multicolumn{7}{c}{ No. (\%) of isolates by ECVs } & No. (\%) of isolates by CBPs \\
\cline { 2 - 7 } & range & $\mathrm{MIC}_{50}$ & $\mathrm{WT}$ & Non-WT & $\mathrm{S}$ & $\mathrm{I}$ & $\mathrm{R}$ \\
\hline $\begin{array}{c}\text { C. guilliermondii (27) } \\
\text { Fluconazole }\end{array}$ & $2->256$ & 4 & $23(85.2)$ & $4(14.8)$ & & & \\
Itraconazole & $0.25->16$ & 0.5 & $24(88.9)$ & $3(11.1)$ & & & \\
Voriconazole & $0.03-8$ & 0.12 & $25(92.6)$ & $2(7.4)$ & & & \\
Posaconazole & $0.06-2$ & 0.25 & $25(92.6)$ & $2(7.4)$ & & & \\
Micafungin & $0.25-1$ & 0.5 & $27(100)$ & $0(0)$ & $27(100)$ & $0(0)$ & $0(0)$ \\
Caspofungin & $0.12-1$ & 0.5 & $27(100)$ & $0(0)$ & $27(100)$ & $0(0)$ & $0(0)$ \\
Anidulafungin & $0.5-4$ & 2 & $27(100)$ & $0(0)$ & $26(96.3)$ & $1(3.7)$ & $0(0)$ \\
Amphotericin B & $0.25-2$ & 0.5 & $27(100)$ & $0(0)$ & & & \\
\hline
\end{tabular}

\begin{tabular}{ccccccccc} 
C. fermentati (12) & & & & & & & \\
Fluconazole & $2-8$ & 4 & $12(100)$ & $0(0)$ & & & \\
Itraconazole & $0.25-0.5$ & 0.25 & $12(100)$ & $0(0)$ & & & \\
Voriconazole & $0.06-0.25$ & 0.12 & $12(100)$ & $0(0)$ & & & \\
Posaconazole & $0.03-0.25$ & 0.12 & $12(100)$ & $0(0)$ & & & \\
Micafungin & $0.25-1$ & 0.5 & $12(100)$ & $0(0)$ & $12(100)$ & $0(0)$ & $0(0)$ \\
Caspofungin & $0.25-1$ & 0.5 & $12(100)$ & $0(0)$ & $12(100)$ & $0(0)$ & $0(0)$ \\
Anidulafungin & $0.5-2$ & 1 & $12(100)$ & $0(0)$ & $12(100)$ & $0(0)$ & $0(0)$ \\
Amphotericin B & $0.5-2$ & 1 & $12(100)$ & $0(0)$ & & & \\
\hline
\end{tabular}

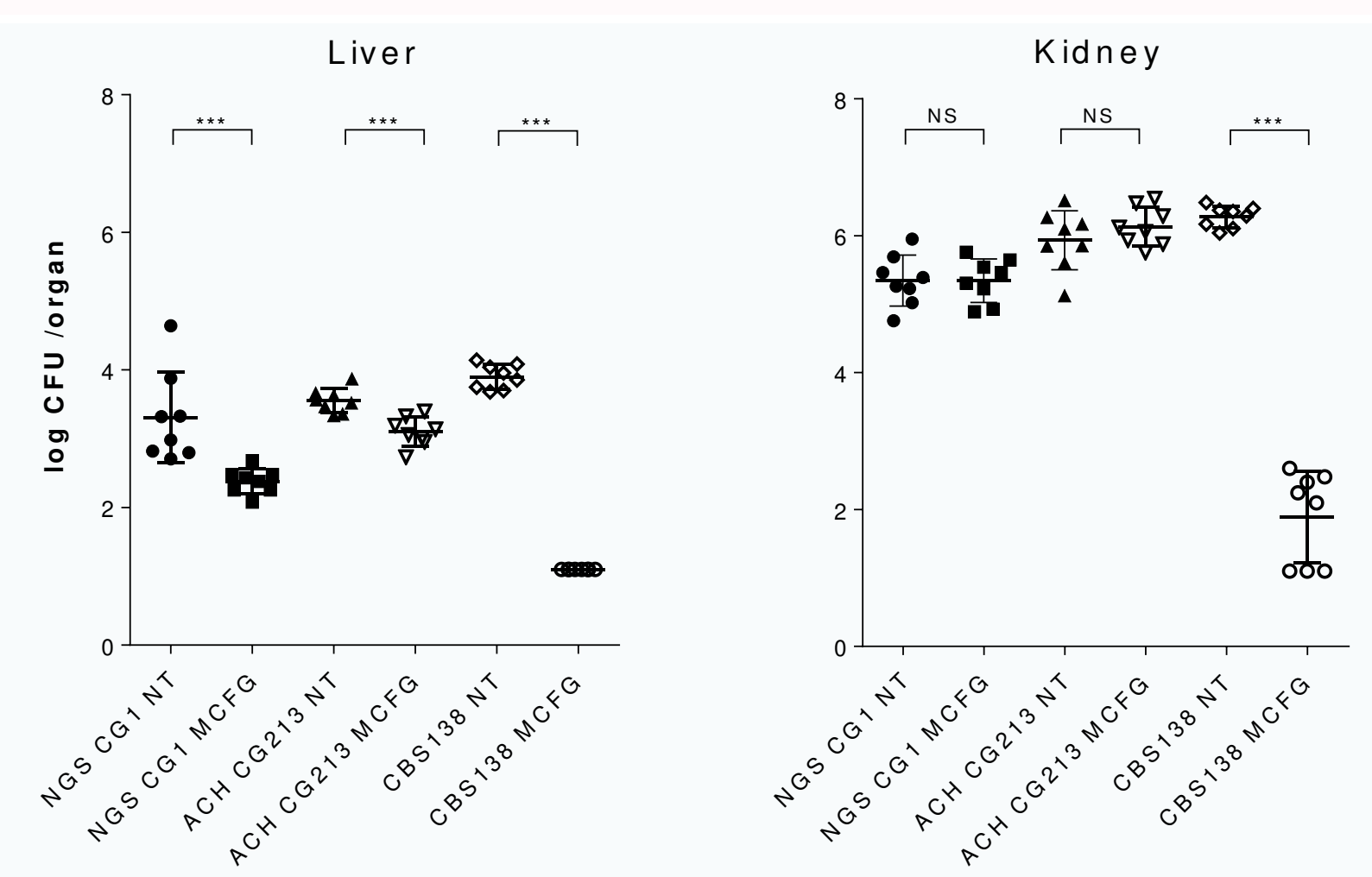

Figure 2. Treatment efficacy of micafungin against $C$. guilliermondii clinical isolates and a $C$. glabrata wild-type strain in a mouse model of disseminated candidiasis. Immunocompromised mice were inoculated with $C$. guilliermondii NGSCG1, C. guilliermondii ACHCG213, and C. glabrata CBS138. Micafungin was administered at $4 \mathrm{mg} / \mathrm{kg} /$ day intraperitoneally for seven consecutive days. The number of cells recovered from the liver and bilateral kidneys is indicated for individual mice in the plots. NT indicates no treatment. Asterisks and NS indicate statistically significant differences $(* * * P<0.001)$ and no significance $(P>0.05)$, respectively. 